\title{
A Eficiência do Ensino de Lógica de Programação na Modalidade a Distância
}

\author{
Emerson A. Carvalho ${ }^{1,2}$ Fábio J. Alves ${ }^{1}$ \\ ${ }^{1}$ IFSULDEMINAS - Campus Machado \\ Rodovia Machado - Paraguaçu - KM 3 - Santo Antônio - Machado - MG - Brasil \\ ${ }^{2}$ Departamento de Engenharia Elétrica \\ Universidade Federal de Itajubá (UNIFEI) - Itajubá - MG - Brasil \\ \{emerson.carvalho, fabio.alves\} @ifsuldeminas.edu.br
}

\begin{abstract}
The Distance Learning has been growing up and expanding the possibilities of teaching. However, due to its nature, the quality of the teaching offered in this approach is questioned, especially when the subjects are complex. The teaching and learning of programming logic is considered a complex subject, with high dropout rates and low performance in related classes. This work aims to evaluate the teaching and learning efficiency of programming logic in EAD courses. Our results indicate that, following appropriate methodologies and making appropriate use of the available technologies for Distance Learning, the average performance and the number of students with a high proficiency level in programming logic classes may be higher than in face-to-face courses.
\end{abstract}

Resumo. A Educação a Distância (EAD) vem crescendo e expandindo as possibilidades de ensino. No entanto, devido a sua natureza, questiona-se a qualidade do ensino nessa modalidade, especialmente em relação a conteúdos complexos. O ensino e aprendizagem da lógica de programação é considerado um tópico complexo, com altos índices de evasão e baixo desempenho em disciplinas correlatas. Esse trabalho objetiva avaliar a eficiência do ensino e aprendizagem da lógica de programação em disciplinas EAD. Nossos resultados indicam que, seguindo uma metodologia adequada e fazendo uso apropriados das tecnologias disponíveis, o desempenho médio e a quantidade de alunos com índice alto de aproveitamento em disciplinas de lógica de programação na modalidade EAD podem ser maiores que em disciplinas estritamente presenciais.

\section{Introdução}

Acredita-se que somente através de uma educação de qualidade, não restringindo a educação formal e profissional, seja possível melhorar a qualidade de vida das pessoas e, consequentemente, o desenvolvimento de um país. As modalidades de ensino vem se desenvolvendo ao longo dos anos e, apesar de suas diversas metodologias e abordagens, podemos separar a educação formal em duas modalidades principais, a Educação Presencial e a $\mathrm{EAD}^{1}$.

A EAD é caracterizada pela separação espaço/tempo entre professor e alunos. Utiliza as Tecnologias da Informação e Comunicação (TICs) como ferramenta de mediação

\footnotetext{
${ }^{1}$ Podemos ter ainda variações entre essas modalidades, como, por exemplo, as modalidades semipresenciais, podendo predominar uma ou outra abordagem.
} 
VII Congresso Brasileiro de Informática na Educação (CBIE 2018)

Anais do XXIV Workshop de Informática na Escola (WIE 2018)

do ensino/aprendizagem, tendo sua evolução diretamente ligada aos avanços dessas tecnologias [Mill and Torres 2013]. As principais características da EAD para os discentes são a possibilidade de focar no tema de interesse que deseja aprender, encontrar o próprio ritmo de estudo, utilizar diversos meios de aprendizagem e a valorização do conhecimento prévio [ABED 2015].

Considerada um avanço na educação, a EAD aumentou a capacidade do sistema educacional em formar, capacitar e especializar, a partir do momento em que as TICs passaram a oferecer melhores ferramentas de apoio ao processo de ensino e aprendizagem [Arieira et al. 2009]. A Internet é uma das grandes responsáveis pela evolução da EAD, a partir do crescimento das redes de comunicação de dados, a expansão dos sinais ofertados pelas operadoras e a redução nos custos de acesso, permitiu a interação entre discentes e docentes em tempo real e o uso de ferramentas interativas, tais como: áudio, vídeo, chats, infográficos, simuladores etc, além de ser fonte de um grande acervo de informações acadêmicas, tais como: apostilas, monografias, dissertações, teses, livros, cursos etc [Mill and Torres 2013].

Incentivadas pelo Ministério da Educação (MEC), várias instituições de ensino oferecem cursos na modalidade EAD em diversos níveis, formação inicial e continuada, técnico, graduação e pós-graduação. Segundo os últimos dados do Censo da Educação Superior ${ }^{2}$, a participação da modalidade no percentual de novos alunos aumentou de 10,8\% em 2006 para 28,2\% em 2016. Uma pesquisa da Associação Brasileira de Mantenedoras de Ensino Superior (ABMES) ${ }^{3}$ prevê que, caso essa tendência de crescimento nas matrículas EAD no ensino superior se mantenha, em 2023 o número de matrículas EAD nas universidades particulares será maior que o de matrículas em cursos presenciais.

A crescente oferta de cursos na modalidade EAD levanta uma preocupação em relação a qualidade da formação oferecida. A qualidade pode ser medida a partir de diversos fatores: recursos oferecidos pelas instituições, relações entre docentes e discentes, conhecimento dos discentes e docentes para lidar com as TICs e metodologias EAD, utilização efetiva dos Ambientes Virtuais de Aprendizagem (AVAs), e ainda um alinhamento de expectativas e responsabilidades por partes de todos os envolvidos no processo de ensino e aprendizagem [Arieira et al. 2009].

A crescente demanda por profissionais de informática fez com que houvesse uma grande oferta de cursos de computação em ambas as modalidades, EAD e presencial. Dentre as complexas disciplinas oferecidas, a lógica de programação é considerada um dos tópicos mais importantes para a formação de um bom profissional da área de computação, por desenvolver habilidades essenciais para um bom rendimento profissional e acadêmico. Entretanto, as disciplinas de lógica, algoritmo e programação são consideradas complexas de ensinar e aprender [Zschaler et al. 2014], e as dificuldades encontradas por parte dos alunos no decorrer dos estudos e práticas levam a altos índices de evasão e reprovação nessas disciplinas [Piekarski et al. 2015, Gomes et al. 2008]. Algumas pesquisas recentes indicam que os índices de evasão em cursos superiores da área de computação podem variar de 56\% [Palmeira and Santos 2014, Souza et al. 2015] a 70\%

\footnotetext{
${ }^{2}$ Sinopses Estatísticas da Educação Superior 2016 (http://portal.inep.gov.br/web/guest/sinopsesestatisticas-da-educacao-superior).

${ }^{3}$ Um ano do Decreto da EAD - O impacto da educação a distância na expansão do ensino superior brasileiro (https://abmes.org.br/abmes-pesquisas/categoria/345/pesquisas).
} 
VII Congresso Brasileiro de Informática na Educação (CBIE 2018)

Anais do XXIV Workshop de Informática na Escola (WIE 2018)

[Florencio 2015].

O docente é considerado, em conjunto com outros fatores, o principal fator crítico para o sucesso dos alunos em cursos de computação [Andrade et al. 2015]. Dessa forma, o ensino e aprendizagem de programação vem sendo tema de diversas pesquisas que buscam encontrar alternativas que auxiliem os estudantes na compreensão dos principais conceitos relacionados à programação de computadores [da Silva et al. 2015]. Para um bom aprendizado, os alunos precisam desenvolver uma série de conhecimentos (matemático, computacional, sintaxe e semântica das linguagens), habilidades (capacidade de corrigir erros e saber relacionar entradas e saídas de um código), atitudes (reflexão sobre os possíveis erros e prática de programação) e competências (criatividade e capacidade de abstração) [Souto and Tedesco 2017].

Em busca do desenvolvimento das habilidades necessárias para um bom desempenho em programação, professores e pesquisadores desenvolveram diferentes abordagens de ensino, tais como: o uso de automação (Arduino) e programação visual [Santos et al. 2015]; robótica [Almeida et al. 2017]; formulação de arquiteturas pedagógicas para aprendizagem de programação [Marques et al. 2016]; aprendizagem baseada em exemplos, fundamentados na dimensão indutiva-dedutiva [Nagano 2016]; utilização de modelos lúdicos e jogos (gamification) [Barbosa et al. 2017, Rodrigues et al. 2017, Santiago and Kronbauer 2016, Scaico and Scaico 2016]; abordagens fundamentadas no pensamento computacional [Zanetti et al. 2016]; abordagens utilizando manipulação de imagens [Ribeiro et al. 2017, Araújo et al. 2017]; aprendizagem baseada em problemas (PBL) [Meira and Borges 2017]; e a criação de ambientes dedicados ao ensino de programação [Amaral et al. 2017]. Um mapeamento sistemático sobre metodologias e ferramentas de apoio ao ensino de programação pode ser visto em [Blatt et al. 2017].

A maioria das abordagens propostas $(81 \%)$ para melhorar o processo de ensino e aprendizagem de programação são desenvolvidas para cursos oferecidos na modalidade presencial, embora façam uso de TICs [da Silva et al. 2015]. Sendo o professor um fator crítico para o desempenho dos alunos nas disciplinas de programação e considerando a separação espaço/tempo entre professor e alunos em ambientes EAD, este trabalho teve o objetivo de avaliar a efetividade das ferramentas e metodologias EAD comumente e amplamente utilizadas no ensino da lógica de programação. A avaliação dessa efetividade foi realizada analisando e comparando o desempenho dos estudantes em disciplinas similares ofertadas nas duas modalidades, EAD e presencial.

\section{Métodos}

O trabalho fundamentou-se numa análise quantitativa do desempenho dos alunos em disciplinas introdutórias de lógica de programação. As disciplinas selecionadas fazem parte da matriz curricular de cursos técnicos em informática nas modalidades presencial, curso técnico integrado ao ensino médio, e EAD, curso subsequente ao ensino médio. As ementas das disciplinas são semelhantes, tendo em comum fundamentos básicos relacionados ao ensino de programação, tais como: lógica de programação, algoritmos, estruturas condicionais e de controle e linguagem de programação C. Foram usados dados de desempenho dos alunos de diversas turmas, espalhadas em todos os semestres dos anos de 2016 e 2017 e ministradas por professores diferentes. O objetivo foi analisar diferentes turmas sob diferentes metodologias (professores) de ensino. Apesar da possibilidade de diferen- 
VII Congresso Brasileiro de Informática na Educação (CBIE 2018)

Anais do XXIV Workshop de Informática na Escola (WIE 2018)

tes professores aplicarem metodologias distintas, algumas características são mantidas, para ambas as modalidades, como práticas institucionais, pois estão presentes nos Projetos Pedagógicos dos cursos.

As disciplinas presenciais são conduzidas, em linhas gerais, seguindo as seguintes abordagens de ensino: 1) aulas teóricas expositivas em sala de aula; 2) aulas práticas em laboratório, com um aluno por computador; 3) aulas semanais de reforço com o professor, ficando a critério do aluno participar das mesmas, apesar da solicitação por parte do professor; 4) monitoria de ensino com monitores selecionados, participação também a critério do aluno e 5) sugestão de leitura do referencial teórico básico (livros textos) da disciplina. O processo avaliativo e de prática das disciplinas presenciais são constituídos de: 1) avaliações teóricas e práticas, 2) trabalhos teóricos e/ou práticos individuais e/ou em grupo e 3) atividades, avaliativas ou não, supervisionadas durante a execução das aulas/reforços/monitorias.

As disciplinas EAD são conduzidas, em linhas gerais, seguindo as seguintes abordagens de ensino: 1) aulas on-line em vídeo, produzidas pelo próprio professor, disponíveis semanalmente via AVA (Moodle); 2) outros recursos on-line (vídeos, textos, podcasts etc) disponibilizados semanalmente por meio do AVA; 3) tutoria de ensino a distância, conduzida diariamente por tutor capacitado através do AVA; 4) tutoria de ensino presencial, conduzida semanalmente por tutor capacitado nos laboratórios dos pólos de apoio presencial; 5) chats semanais com o professor e tutores a distância para esclarecimento de dúvidas e 6) sugestão de leitura do referencial teórico básico (livros textos e apostila) da disciplina. O processo avaliativo e de prática das disciplinas EAD são constituídos de: 1) avaliações semanais teóricas e práticas, aplicadas por meio do AVA 2) trabalhos semanais teóricos e/ou práticos desenvolvidos semanalmente por meio do AVA; 3) participação em fóruns semanais de discussão, retirada de dúvidas ou resolução de problemas, disponibilizados semanalmente para cada tema e 4) avaliações teóricas e práticas supervisionadas, essas sendo realizadas presencialmente nos pólos de apoio presencial.

Caracterizadas as disciplinas cujos dados serviram de base para nosso estudo, utilizamos as notas finais obtidas pelos alunos como critério para avaliar o desempenho dos mesmos. Foram consideradas as notas de todas as atividades realizadas, tanto para as turmas presenciais quando para as turmas EAD. Em relação às atividades avaliativas das disciplinas EAD, 80\% delas foram realizadas a distância (questionários, atividades práticas, fóruns, resolução de problemas etc) e $20 \%$ foram realizas em atividades presenciais. Portanto, $80 \%$ da nota foi avaliada por meio de atividades em ambiente não controlado, propenso à fraudes ('colas'). Devido a esse motivo, os $20 \%$ da nota atribuídos a partir das avaliações de atividades presenciais, considerado um ambiente controlado, teve peso maior na composição da nota final utilizada nesse estudo. Dessa forma, as atividades a distância tiveram peso 1, enquanto as atividades presenciais tiveram peso 4. Dessa forma, fizemos com que a nota final fosse a média das avaliações presenciais e a distância. Consideramos que, a partir dessa abordagem, aproximamos os critérios de avaliação entre as duas modalidades.

Em relação as amostras selecionadas para composição dos dados analisados, utilizamos apenas as notas dos alunos que não evadiram das suas respectivas disciplinas. Para as disciplinas presenciais, consideramos os alunos que não trancaram a disciplina e obtiveram frequência às aulas maior que $75 \%$. Para as disciplinas $\mathrm{EAD}$, como não 
VII Congresso Brasileiro de Informática na Educação (CBIE 2018)

Anais do XXIV Workshop de Informática na Escola (WIE 2018)

havia critério de presença, consideramos os alunos que realizaram pelo menos $75 \%$ das atividades avaliativas propostas ou alcançaram nota maior que 6 pontos (suficientes para aprovação, independente da quantidade de atividades realizadas). Essa estratégia visou, para ambas as modalidades, eliminar das amostras aqueles alunos que, direta ou indiretamente, evadiram das disciplinas. Nas disciplinas presenciais tivemos $2 \%$ de evasão, restando uma amostra de 142 alunos, já nas disciplinas EAD a evasão foi de 33\%, com amostra final de 117 alunos.

\section{Resultados e Discussões}

Nossa primeira análise se deu a partir da comparação da quantidade de alunos, para cada modalidade, em relação à faixa de notas obtidas. Para isso, definimos 5 conceitos para segmentar as notas em 5 faixas que consideramos, já a partir dos dados obtidos, relevantes para nossa análise. A Tabela 1 mostra a segmentação da nota possível (10) em 5 conceitos/faixas.

\begin{tabular}{|c|c|}
\hline Conceito & Nota $(\mathbf{x})$ \\
\hline A & $8.0 \leq x \leq 10$ \\
\hline B & $6.0 \leq x<8.0$ \\
\hline C & $4.0 \leq x<6.0$ \\
\hline D & $2.0 \leq x<4.0$ \\
\hline E & $0.0 \leq x<2.0$ \\
\hline
\end{tabular}

Tabela 1. Conceitos por faixa de valores para as notas

Conforme pode ser visto no gráfico da Figura 1, 44\% dos alunos das disciplinas EAD obtiveram conceito A, enquanto apenas $22 \%$ dos alunos das disciplinas presenciais obtiveram o mesmo conceito, um percentual $50 \%$ menor nas turmas presenciais. Essa situação se inverte em relação ao conceito $\mathrm{B}$, ainda que em uma proporção bem menor (14.5\% menor nas turmas EAD), com $41 \%$ dos alunos das disciplinas presenciais alcançando esse nível de desempenho, em relação a 35\% dos alunos das disciplinas EAD. Considerando que as disciplinas requeriam, em ambas as modalidades, um rendimento maior ou igual a 6 pontos (conceito A ou B) para aprovação dos alunos, 79\% dos alunos das disciplinas EAD foram aprovados, em relação a $63 \%$ dos alunos das disciplinas presenciais, um percentual $20 \%$ menor nas turmas presenciais.

Esses índices de aprovação (63\% presencial e 79\% EAD) são bem superiores aos índices apontados por [Mourão 2017] ${ }^{4}$ que variam de $40 \%$ a $45 \%$ enquanto usando metodologias tradicionais para o ensino presencial, e próximos aos índices, que variam de 67 a $72 \%$ enquanto usando metodologias ativas baseada em problemas e coding dojo $^{5}$.

Consequentemente, o percentual de alunos das disciplinas presenciais que obtiveram notas inferiores a média requerida para aprovação (conceitos $\mathrm{C}, \mathrm{D}$ ou $\mathrm{E}$ ) foi maior, $26 \%$ e $11 \%$ para os níveis C e D respectivamente. Esse percentual é bem menor em relação as disciplinas EAD, 16\% e 5\% para os níveis C e D respectivamente. As disciplinas

\footnotetext{
${ }^{4}$ A pesquisa avalia alunos de cursos superiores de instituições públicas e privadas. Avaliando o índice de reprovação antes e depois da aplicação de metodologias ativas.

${ }^{5}$ Atividade que reúne programadores para programar e solucionar problemas em grupo, compartilhando conhecimentos e favorecendo o aprendizado voluntário e colaborativo.
} 
VII Congresso Brasileiro de Informática na Educação (CBIE 2018)

Anais do XXIV Workshop de Informática na Escola (WIE 2018)

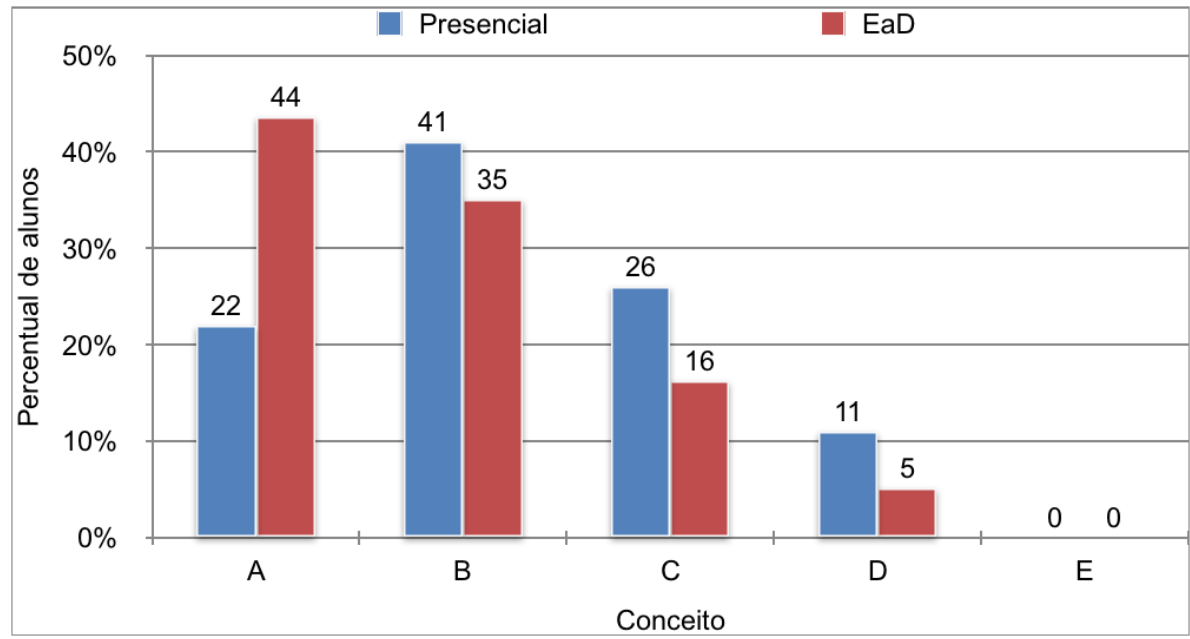

Figura 1. Percentual de alunos por conceito obtido

EAD tem percentual de alunos $38.5 \%$ e $54.5 \%$ menores para os níveis C e D respectivamente. Não houveram alunos, em nenhuma das modalidades, com conceito E.

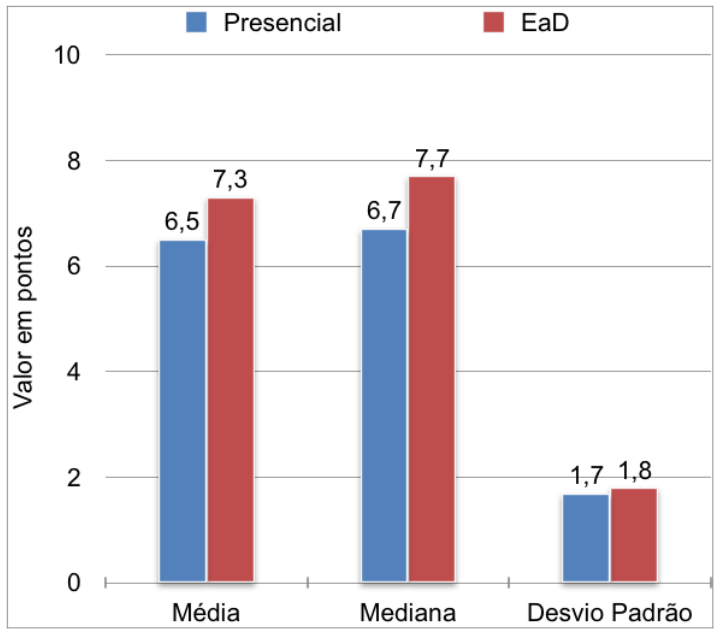

(a)

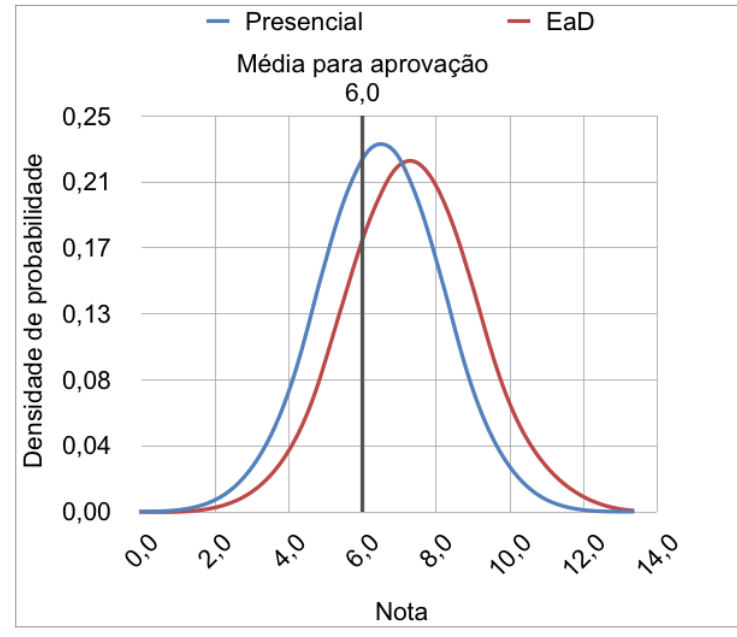

(b)

Figura 2. (a) Medidas de tendência central e (b) Distribuição normal

Considerando as medidas de tendência central, Figura 2 (a), podemos observar que, apesar das disciplinas EAD apresentarem melhores valores, a média aritmética e a mediana das notas não são muito distantes em relação as duas modalidades, aproximadamente $11 \%$ menor para as disciplinas presenciais, com o desvio padrão ligeiramente menor nas disciplinas presenciais. As médias das notas nas modalidades se assemelham as médias obtidas por [Amaral et al. 2017] (7.0 presencial e 7.1 EAD) em turmas onde foi avaliado o desempenho dos alunos na modalidade presencial em comparação com uma abordagem assistida por ferramenta EAD, ainda que nossa média de desempenho na modalidade EAD (7.3) foi um pouco superior, enquanto a média na modalidade presencial (6.5) foi relativamente inferior. No entanto, se considerarmos a baixa média das notas obtida por [Coutinho et al. 2017] ao analisar o desempenho de 5 turmas na disciplina de lógica de programação, que foi de 4.7, os resultados que obtivemos, para ambas as moda- 
VII Congresso Brasileiro de Informática na Educação (CBIE 2018)

Anais do XXIV Workshop de Informática na Escola (WIE 2018)

lidades, foram consideravelmente superiores.

Os desvios padrão, no entanto, foram considerados alto, visto que, para ambas as modalidades, a média aritmética subtraída de um (1) desvio padrão levaria a um valor inferior a média necessária (6 pontos) para a aprovação dos alunos, o que diminui o intervalo de confiança da parte inferior à média, principalmente para as disciplinas presenciais. No entanto, ao realizar a distribuição normal das medidas centrais exibidas na Figura 2 (a), obtemos as curvas normais presentes na Figura 2 (b). Calculando as áreas sob as curvas que estão acima da média requerida para aprovação (6 pontos), obtemos a probabilidade de aprovação dos alunos em cada modalidade. A probabilidade de aprovação é de $61.5 \%$ para as disciplinas presenciais e $76.5 \%$ para as disciplinas EAD. Essas probabilidades estão muitos próximas aos percentuais de aprovação, conceitos A ou B da Figura 1, o que corrobora para que esses valores sejam considerados confiáveis para mensurar o desempenho das turmas, apesar dos desvios padrões serem considerados altos.

\section{Conclusões}

O ensino e aprendizagem da lógica de programação é tema recorrente em diversas pesquisas que buscam alternativas para melhorar a eficiência desse processo. A complexidade envolvida no aprendizado da programação de computadores, bem como os altos índices de evasão e reprovação em disciplinas relacionadas a esse tema, motivam professores e pesquisadores a buscarem por novas práticas pedagógicas, ferramentas tecnológicas de apoio e metodologias que possam reverter esse quadro.

No ensino presencial da programação podemos experimentar diversas metodologias e práticas pedagógicas. Utilizar as melhores ferramentas tecnológicas de apoio ao processo de ensino e aprendizagem, acompanhar de perto (tempo e espaço) o desenvolvimento das atividades práticas propostas e monitorar regularmente a motivação, o desempenho e as dificuldades gerais e específicas dos alunos.

No que tange a EAD, temos um agravante que dificulta o monitoramento da eficiência do processo de ensino e aprendizagem da lógica de programação. O fato do professor estar separado dos seus alunos, em relação ao tempo e espaço, requer medidas que permitam que as práticas básicas necessárias ao ensino da lógica de programação possam ser executadas.

Para alcançar um boa eficiência e melhores índices de aprendizado e aproveitamento no ensino EAD da lógica de programação é necessário mitigar os problemas que possam surgir em decorrência dos seguintes fatores (não restrito a esses): 1) assiduidade nos conteúdos (vídeos, textos, fóruns e atividades propostas); 2) qualidade das respostas das atividades práticas, especialmente aquelas que envolvem o desenvolvimento de algoritmos e códigos fonte; 3) feedback apropriado e pontual em relação às dificuldades percebidas através da leitura e correção das atividades práticas; 4) estímulo em participar de fóruns e analisar/ler algoritmos e códigos dos colegas de turma; 5) apresentação e incentivo na busca de outras fontes de recursos (vídeos, textos e códigos) relacionados a cada tema e 6) análise de desempenho por tema, com consequente reforço dos temas onde é percebido baixo aproveitamento por parte dos alunos.

Planejadas adequada e cuidadosamente, e mediadas por AVAs robustos, que nos permite realizar o acompanhamento de variáveis que nos dão indicadores dos fatores 
VII Congresso Brasileiro de Informática na Educação (CBIE 2018)

Anais do XXIV Workshop de Informática na Escola (WIE 2018)

acima citados, disciplinas de lógica de programação na modalidade EAD podem ter uma eficiência no processo de ensino e aprendizagem igual ou superior a disciplinas presenciais equivalentes, levando a um número maior de alunos aprovados. Considerando aqueles alunos mais assíduos, que provavelmente são os que exploram todos os materiais, possivelmente mais de uma vez, acreditamos que a natureza dos conteúdos EAD (podem ser vistos a qualquer hora, em qualquer lugar e quantas vezes forem necessárias), possibilita que um percentual maior de alunos, $50 \%$ em relação ao ensino presencial, de acordo com nosso estudo, alcance um nível A de excelência.

Essa eficiência na EAD é possível em grande parte graças às ferramentas tecnológicas de diversas natureza disponíveis atualmente, tais como ferramentas para criação e edição de vídeos, plataformas de hospedagem, AVAs com indicadores diversos, ferramentas para edição colaborativa de código, ferramentas para hospedagem e compartilhamento de código, editores e compiladores de código amigáveis. Pela experiência vivida na condução dessas disciplinas, não podemos negar que é mais trabalhoso conduzir uma disciplina a distância apoiada por essas ferramentas. Ainda precisamos considerar o fato de que há mais profissionais envolvidos no processo como um todo. No entanto, além de todas as vantagens inerentes ao fator tempo e espaço, nossos resultados indicam que disciplinas de programação presenciais poderiam se beneficiar do uso de ferramentas EAD de apoio em diversos aspectos (disseminação de materiais, atividades em grupo, análises de desempenho etc) de forma a alcançar maior engajamento e aprendizagem por parte dos alunos.

Os próximos passos em nossas pesquisas relacionadas ao ensino e aprendizagem da lógica de programação será aplicar técnicas e ferramentas EAD em turmas presenciais, analisando a melhoria de desempenho que poderia ser alcançada em relação a turmas estritamente presenciais.

\section{Referências}

ABED, A. B. d. E. a. D. (2015). Educação aberta e a distância no Brasil - o estado da arte. In: ABED 20 anos. A educação a distância no Brasil: presente passado futuro. Plano B Editorial, São Paulo, SP.

Almeida, T., Netto, J. F., da Silva, R., and Custódio, T. (2017). Laboratório remoto de robótica como elemento motivador para a aprendizagem de programação. In Brazilian Symposium on Computers in Education (Simpósio Brasileiro de Informática na Educação-SBIE), volume 28, page 665.

Amaral, E., Camargo, A., Gomes, M., Richa, C. H., and Becker, L. (2017). Algo+ uma ferramenta para o apoio ao ensino de algoritmos e programação para alunos iniciantes. In Brazilian Symposium on Computers in Education (Simpósio Brasileiro de Informática na Educação-SBIE), volume 28, page 1677.

Andrade, M., Neto, N., de Sousa, D. F., Chaves, R., and Carvalho, G. (2015). Análise dos fatores críticos para o sucesso de cursos de graduação em computação: um estudo de caso na região amazônica. In Anais do Workshop de Informática na Escola, volume 21, page 574 .

Araújo, L. G., Bittencourt, R., and dos Santos, D. M. B. (2017). Uma abordagem contextualizada para o ensino de programação na educação profissional em informática. In 
VII Congresso Brasileiro de Informática na Educação (CBIE 2018)

Anais do XXIV Workshop de Informática na Escola (WIE 2018)

Anais dos Workshops do Congresso Brasileiro de Informática na Educação, volume 6, page 1021.

Arieira, J. d. O., Dias-Arieira, C. R., Alves Fusco, J. P., Benedito Sacomano, J., and Odette de Pauli Bettega, M. (2009). Avaliação do aprendizado via educação a distância: a visão dos discentes. Ensaio: avaliação e políticas públicas em Educação, 17(63).

Barbosa, B., Silva, S., and Sousa, B. (2017). Tri-logic proposta lúdica gamificada para o ensino e aprendizagem da lógica de programação com o uso da mineração de dados como ferramenta de auxílio ao professor. In Brazilian Symposium on Computers in Education (Simpósio Brasileiro de Informática na Educação-SBIE), volume 28, page 1754.

Blatt, L., Becker, V., and Ferreira, A. (2017). Mapeamento sistemático sobre metodologias e ferramentas de apoio para o ensino de programação. In Anais do Workshop de Informática na Escola, volume 23, page 815.

Coutinho, E. F., de Lima, E. T., and Santos, C. C. (2017). Um panorama sobre o desempenho de uma disciplina inicial de programaçao em um curso de graduaçao. Revista Tecnologias na Educaç ao, 19(9).

da Silva, T. R., Medeiros, T., Medeiros, H., Lopes, R., and Aranha, E. (2015). Ensinoaprendizagem de programação: uma revisão sistemática da literatura. Revista Brasileira de Informática na Educação, 23(1).

Florencio, F. G. (2015). Evasão no curso de licenciatura em computação da universidade de brasília. Monografia (Licenciatura em Computação), Universidade de Brasília UnB, Brasília / DF, Brasil.

Gomes, A., Henriques, J., and Mendes, A. (2008). Uma proposta para ajudar alunos com dificuldades na aprendizagem inicial de programação de computadores. Educação, Formação \& Tecnologias-ISSN 1646-933X, 1(1):93-103.

Marques, G., Almeida, R., Junior, R. M., and Tavares, O. (2016). Ambiente para aprendizagem de programação fundamentado em arquiteturas pedagógicas. In Brazilian Symposium on Computers in Education (Simpósio Brasileiro de Informática na EducaçãoSBIE), volume 27, page 1275 .

Meira, M. and Borges, M. (2017). Aprendizagem de linguagem de programação com metodologia pbl em competições científicas com robocode. In Anais do Workshop de Informática na Escola, volume 23, page 195.

Mill, D. and Torres, M. A. G. (2013). Educação a Distância: Um Guia para o Estudante Virtual. Editora Montes Claros, Montes Claros, MG.

Mourão, A. (2017). Uma proposta da eficiência do uso da metodologia ativa baseada em problemas, utilizando dojo de programação, aplicada na disciplina de lógica de programação. In Anais do Workshop de Informática na Escola, volume 23, page 667.

Nagano, L. (2016). Ensino de lógica de programação baseado na indução-dedução através de exemplos. In Brazilian Symposium on Computers in Education (Simpósio Brasileiro de Informática na Educação-SBIE), volume 27, page 1352.

Palmeira, L. B. and Santos, M. P. (2014). Evasão no bacharelado em ciência da computação da universidade de brasília: análise e mineração de dados. Monografia (Ba- 
VII Congresso Brasileiro de Informática na Educação (CBIE 2018)

Anais do XXIV Workshop de Informática na Escola (WIE 2018)

charelado em Ciência da Computação), Universidade de Brasília - UnB, Brasília / DF, Brasil.

Piekarski, A. E., Miazaki, M., Hild, T., Mulati, M. H., and Kikuti, D. (2015). A metodologia das maratonas de programação em um projeto de extensão: um relato de experiência. In Anais dos Workshops do Congresso Brasileiro de Informática na Educação, volume 4, page 1246.

Ribeiro, A. L., Carvalho, I. C., Araújo, L. G., and Bittencourt, R. (2017). Computação com mídias na aprendizagem de programação orientada a objetos em um curso técnico de informática. In Anais dos Workshops do Congresso Brasileiro de Informática na Educação, volume 6, page 1031.

Rodrigues, L. C., Nogueira, G. C., and Queiroga, A. (2017). Experiências no ensino de programação orientada a objetos: Robocode, greenfoot e jogos de tabuleiro no ensino superior. In Anais do Workshop de Informática na Escola, volume 23, page 598.

Santiago, A. D. and Kronbauer, A. (2016). Um modelo lúdico para o ensino de conceitos de programação de computadores. In Brazilian Symposium on Computers in Education (Simpósio Brasileiro de Informática na Educação-SBIE), volume 27, page 420.

Santos, A., Gorgônio, A., Lucena, A., and Gorgônio, F. (2015). A importância do fator motivacional no processo ensino-aprendizagem de algoritmos e lógica de programação para alunos repetentes. In WEI-Workshop sobre Educação em Computação, pages 110 .

Scaico, A. and Scaico, P. (2016). Uso de jogos em cursos introdutórios de programaçãouma revisão sistemática. In Brazilian Symposium on Computers in Education (Simpósio Brasileiro de Informática na Educação-SBIE), volume 27, page 549.

Souto, M. and Tedesco, P. (2017). Uma revisão sistemática da literatura sobre conhecimentos, habilidades, atitudes e competências desejáveis para auxiliar a aprendizagem de programação. In Anais dos Workshops do Congresso Brasileiro de Informática na Educação, volume 6, page 1162.

Souza, O. S., Morais, P. S., and Júnior, F. C. S. (2015). Um estudo sobre a evasão no curso de licenciatura em informática do ifrn-campus natal-zona norte. In XXIII Workshop sobre Educação em Computação(WEI).

Zanetti, H., Borges, M., and Ricarte, I. (2016). Pensamento computacional no ensino de programação: uma revisão sistemática da literatura brasileira. In Brazilian Symposium on Computers in Education (Simpósio Brasileiro de Informática na Educação-SBIE), volume 27, page 21.

Zschaler, S., Demuth, B., and Schmitz, L. (2014). Salespoint: A java framework for teaching object-oriented software development. Science of Computer Programming, 79:189-203. 